\title{
DISCRETE FRACTIONAL HADAMARD TRANSFORM
}

\author{
Soo-Chang Pei and Min-Hung Yeh ${ }^{2}$ \\ ${ }^{1}$ Department of Electrical Engineering, National Taiwan University, Taipei, Taiwan, R. O. C. \\ ${ }^{2}$ Department of Computer Information Science, Tamsui Oxford University College, Tamsui, Taipei, Taiwan, R. O. C.
}

\begin{abstract}
Hadamard transform is an important tool in discrete signal processing. In this paper, we define the discrete fractional Hadamard transform which is a generalized one. The development of discrete fractional Hadamard is based upon the same spirit of discrete fractional Fourier transform.
\end{abstract}

\section{Introduction}

Fractional Fourier transform(FRFT) is a generalization of Fourier transform, and its output can have the mixed time and frequency components of signal[1]. Because of the importance of FRFT, the discrete fractional Fourier transform (DFRFT) has become an important issue in recont yeurs. In [2], a DFRFT has been proposed by B. Santhanam and J. H. McClellan. Unfortunately, this DFRFT can not have similar results as those of continuous case. In 1996, Pei and Yeh have found that the DFRFTs with DFT Hermite eigenvectors can provide similar results as continuous case[3][4].

On the other hand, many orthogonal transforms have been successfully used in signal processing. Some typical ones are discrete cosine transform (DCT)[5], discrete Hartley transform (DHT) [5] and Hadamard transform. Until now, the fractional version of DFT[4] and DHT[6] have also been investigated and successfully used in signal processing. But the discrete fractional Hadamard transform was still absent. The goal of this paper is give a definition for the discrete fractional Hadamard transform.

\section{Preliminary}

For the factor of normalization, The DFT kernel is defined as the following way:

$$
\mathrm{F}=\sqrt{\frac{1}{N}}\left[\begin{array}{ccccc}
1 & 1 & \cdots & 1 & 1 \\
1 & W_{N}^{1} & \cdots & W_{N}^{N-2} & W_{N}^{N-1} \\
\vdots & & \ddots & & \vdots \\
1 & W_{N}^{N-2} & \cdots & W_{N}^{(N-2)^{2}} & W_{N}^{(N-1)(N-2)} \\
1 & W_{N}^{N-1} & \cdots & W_{N}^{(N-1)(N-2)} & W_{N}^{(N-1)^{2}}
\end{array}\right]
$$

where $W_{N}=e^{-j \frac{2 \pi}{N}}$. In the history of DFRFT, many DFRFTs have been proposed. The DFRFT in [2] cannot have the similar results[7]. The DFRFT concerned in this paper is based on the eigen decomposition method proposed by Pei and Yeh[3] [4]. The methods in [3][4] use the DFT Hermite eigenvectors to construct DFRF'T kernel matrix. and have similar outputs as the continuous results. In
[3][4], the N-point DFRFT kernels are computed as follows:

$$
\begin{aligned}
\mathbf{F}_{\alpha} & =\sum_{0}^{N-1} e^{-j k \alpha} \mathbf{v}_{k} \mathbf{v}_{k}^{T} \\
& =V\left[\begin{array}{cccc}
1 & & & \\
& e^{-j \alpha} & & \\
0 & & \ddots & \\
& & e^{-j(N-1) \alpha}
\end{array}\right] \mathbf{V}^{T}
\end{aligned}
$$

where $\mathbf{V}=\left[\mathbf{v}_{0}\left|\mathbf{v}_{1}\right| \ldots \mid \mathbf{v}_{N-1}\right] . \mathbf{v}_{k}$ is the the $k$-th order DFT Hermite eigenvector. $\alpha$ indicates the rotation angle of transform in the time-frequency plane. The methods for finding the $k$-th order DFT Hermite eigenvectors have been shown in [3] and [4].

A Hadamard matrix is a symmetric matrix whose elements are the real numbers 1 and $-1[5]$. The rows(and columns) of a Hadamard matrix are mutually orthogonal. The normalized Hadamard matrices of order $2^{n}$, denoted by $\mathbf{H}_{n}$, can be recursively by defining

$$
\mathbf{H}_{1}=\frac{1}{\sqrt{2}}\left[\begin{array}{cc}
1 & 1 \\
1 & -1
\end{array}\right] \quad \mathbf{H}_{n+1}=\frac{1}{\sqrt{2}}\left[\begin{array}{cc}
\mathbf{H}_{n} & \mathbf{H}_{n} \\
\mathbf{H}_{n} & -\mathbf{H}_{n}
\end{array}\right] \quad n \geq 1
$$

In [8], a method for finding the eigenvalues and eigenvectors of Hadamard transform has been proposed. Here we will state it briefly.

Proposition 1 lf $\mathbf{v}_{n, k}$ is an eigenvector of $\mathbf{H}_{n}$ corresponding to the eigenvalue $\lambda$,

$$
\hat{\mathbf{v}}_{n, k}=\left[\begin{array}{c}
\mathbf{v}_{n, k} \\
(-1+\sqrt{2}) \mathbf{v}_{n, k}
\end{array}\right]
$$

will be an eigenvector of $\mathbf{H}_{n+1}$, and it corresponds to the eigenvalue $\lambda$.

Proof : See [8].

Proposition 1 gives us a method for finding the eigenvectors from order $2^{n}$ to order $2^{n+1}$. The initial eigenvector that is an eigenvector of $\mathbf{H}_{1}$ is show as follows:

$$
\left[\begin{array}{c}
1 \\
\sqrt{2}-1
\end{array}\right]
$$

Using Proposition 1 and Eq.(6), only one eigenvector of order $2^{n}$ can be computed. In [8], a sequence of matrices $E_{n}$ are defined to generate a set of orthogonal and complete eigenvectors.

$$
\mathbf{E}_{0}=[-1] \quad \mathbf{E}_{n+1}=\left[\begin{array}{cc}
0 & \mathbf{E}_{n} \\
-\mathbf{E}_{n} & 0
\end{array}\right]
$$


Besides $\mathbf{E}_{k}$, a matrix $\mathbf{P}_{n k}$ is also defined by the direct sum of $2^{n-k}$ copies of $\mathbf{E}_{k}$ 's:

$$
\mathbf{P}_{n k}=\mathbf{E}_{k} \oplus \mathbf{E}_{k} \oplus \cdots \oplus \mathbf{E}_{k}
$$

Then the orthogonal and complete Hadamard eigenvectors $\mathbf{Y}_{\boldsymbol{i}}$ can be obtained:

$$
\mathbf{Y}_{i}=\mathbf{Q}_{i-1} \mathbf{Y}_{i-1} \quad 2 \leq i \leq 2^{n}
$$

where

$$
\mathbf{Q}_{2^{r}(2 k+1)}=\mathbf{P}_{n(r+1)} \quad 0 \leq k
$$

The first eigenvector $\mathbf{Y}_{2}$ is computed by Proposition 1 and Eq.(6). The completeness and orthogonality in $\mathbf{Y}_{i}$ have been proved in [8].

\section{A recursive method for Hadamard eigenvectors and eigenvalues}

Here we will develop a new recursive method, which can be used to compute the Hadamard eigenvectors from order $2^{n}$ to $2^{n+1}$ directly. No auxiliary matrix (such as $\mathbf{E}_{n}$ and $\mathbf{P}_{n}$ in [8]) is required.

Proposition 2 If $\mathbf{v}_{n, k}$ is an eigenvector of $\mathbf{H}_{n}$ corresponding to the eigenvalue $\lambda$,

$$
\hat{\mathbf{v}}_{n, k}=\left[\begin{array}{c}
(1-\sqrt{2}) \mathbf{v}_{n, k} \\
\mathbf{v}_{n, k}
\end{array}\right]
$$

will be an eigenvector of $\mathbf{H}_{n+1}$, and it corresponds to the eigenvalue $-\lambda$.

Proof : The following proof is directly from the definition of eigenvector.

$$
\begin{aligned}
\mathbf{H}_{n+1} \tilde{\mathbf{v}}_{n, k} & =\frac{1}{\sqrt{2}}\left[\begin{array}{cc}
\mathbf{H}_{n} & \mathbf{H}_{n} \\
\mathbf{H}_{n} & -\mathbf{H}_{n}
\end{array}\right]\left[\begin{array}{c}
(1-\sqrt{2}) \mathbf{v}_{n, k} \\
\mathbf{v}_{n, k}
\end{array}\right] \\
& =\frac{1}{\sqrt{2}}\left[\begin{array}{c}
(2-\sqrt{2}) \mathbf{H}_{n} \mathbf{v}_{n, k} \\
-\sqrt{2} \mathbf{H}_{n} \mathbf{v}_{n, k}
\end{array}\right] \\
& =\frac{1}{\sqrt{2}}\left[\begin{array}{c}
2-\sqrt{2} \lambda \mathbf{v}_{n, k} \\
-\sqrt{2} \lambda \mathbf{v}_{n, k}
\end{array}\right]=-\lambda \overline{\mathbf{v}}_{n, k}
\end{aligned}
$$

This proposition has been proved.

Proposition 1 and 2 can give us a recursive method for generating the Hadamard eigenvectors of order $2^{n+1}$ from the eigenvectors of order $2^{n}$. The two initial eigenvectors for this algorithm are:

$$
\mathbf{v}_{1,0}=\left[\begin{array}{c}
1 \\
\sqrt{2}-1
\end{array}\right] \quad \mathbf{v}_{1,1}=\left[\begin{array}{c}
1-\sqrt{2} \\
1
\end{array}\right]
$$

where $\mathbf{v}_{1,0}$ and $\mathbf{v}_{1,1}$ are the eigenvectors of $\mathbf{H}_{1}$. In the following proposition, we will verify the orthogonality and completeness in the generated Hadamard eigenvectors by Propositions 1 and 2.

Proposition 3 The following orthogonality can be easily derived in the Hadamard eigenvectors.

1. $\hat{\mathbf{v}}_{n, k}$ and $\tilde{\mathrm{v}}_{n, l}$ are orthogonal. $\left(0 \leq k, l \leq 2^{n}-1\right)$

2. If $\mathbf{v}_{n, k}$ and $\mathbf{v}_{n, l}$ are orthogonal, $\hat{\mathbf{v}}_{n, k}$ and $\hat{\mathbf{v}}_{n, l}$ will be also orthogonal. $\left(k \neq l, 0 \leq k, l \leq 2^{n-1}\right)$

3. If $\mathbf{v}_{n, k}$ and $\mathbf{v}_{n, l}$ are orthogonal, $\tilde{\mathbf{v}}_{n, k}$ and $\tilde{\mathbf{v}}_{n, l}$ will be also orthogonal. $\left(k \neq l, 0 \leq k, l \leq 2^{n-1}\right)$
Proof: To begin with, the orthogonality in $\hat{\mathbf{v}}_{n, k}$ and $\tilde{\mathbf{v}}_{n, l}$ will be proved.

$$
\begin{aligned}
\hat{\mathbf{v}}_{n, k}^{T} \overline{\mathbf{v}}_{n, l} & =\left[\mathbf{v}_{n, k}^{T}(-1+\sqrt{2}) \mathbf{v}_{n, k}^{T}\right]\left[\begin{array}{c}
(1-\sqrt{2}) \mathbf{v}_{n, l} \\
\mathbf{v}_{n, l}
\end{array}\right] \\
& =(1-\sqrt{2}) \mathbf{v}_{n, k}^{T} \mathbf{v}_{n, l}+(-1+\sqrt{2}) \mathbf{v}_{n, k}^{T} \mathbf{v}_{n, l}=0
\end{aligned}
$$

Then we will prove the orthogonality in $\hat{v}_{n, k}$ and $\hat{\mathbf{v}}_{n, l}$.

$$
\begin{aligned}
\hat{\mathbf{v}}_{n, k}^{T} \hat{\mathbf{v}}_{n, l} & =\left[\mathbf{v}_{n, k}^{T}(-1+\sqrt{2}) \mathbf{v}_{n, k}^{T}\right]\left[\begin{array}{c}
\left(1-\sqrt{2} \mathbf{v}_{n, l}\right. \\
\mathbf{v}_{n, l}
\end{array}\right] \\
& =(1-\sqrt{2}) \mathbf{v}_{n, k}^{T} \mathbf{v}_{n, l}+(-1+\sqrt{2}) \mathbf{v}_{n, k}^{T} \mathbf{v}_{n, l}=0
\end{aligned}
$$

The orthogonality in $\overline{\mathbf{v}}_{n, k}$ and $\tilde{\mathbf{v}}_{n, l}$ can be proved similar to that in $\hat{\mathbf{v}}_{n, k}$ and $\hat{\mathbf{v}}_{n, l}$. []

Using Propositions 1 and 2, each eigenvector $\mathbf{v}_{n, k}$ for order $2^{n}$ can generate two new eigenvectors for order $2^{n+1}$. So the number of Hadamard eigenvectors will be twice as the number of order $2^{n}$. Because the initial case $(n=1)$ is with two eigenvectors in $\mathrm{Eq}(12)$, the number of Hadamard eigenvectors generated by the recursive algorithm is exact $2^{n}$ for order $2^{n}$. Moreover, the orthogonality in the generated eigenvectors has been proved in Proposition 3. So the generated Hadamard eigenvectors are complete and orthogonal.

Proposition 4 The eigenvalues of discrete Hadamard matrix are only 1 and -1 .

Proof: Because $\mathbf{H}_{1}$ has the only two cigenvalues 1 and -1 , the complete Hadamard eigenvectors computed by Proposition 1 and 2 will be only with these two eigenvalues.

In the development of DFRFT, the DFRFT uses the DFT Hermite eigenvectors as its eigenvectors. An important feature in the DFT Hermite eigenvectors is that the $k$-th order Hermite function can have $k$ sign-changes [3][4]. In the following, we will check the sign-changes in the computed Hadamard eigenvectors and use the sign-changes to develop the fractional Hadamard transform.

Proposition 5 If $\mathbf{v}_{n, k}$ is with $k$ sign-changes, the number of sign-changes in $\hat{\mathbf{v}}_{n, k}$ and $\hat{\mathbf{v}}_{n, k}$ are $2 k$ and $2 k+1$. One is with $2 k$, and the other will have $2 k+1$.

Proof : Because the two halves of $\hat{\mathbf{v}}_{n, k}$ and $\overline{\mathbf{v}}_{n, k}$ are directly from $\mathbf{v}_{n, k}$, the sign-changes of $\hat{\mathbf{v}}_{n, k}$ and $\overline{\mathbf{v}}_{n, k}$ are at least $2 k$ and no more than $2 k+1$. The sign-change in the conjunction of the first and second halves of $\hat{\mathbf{v}}_{n, k}$ and $\tilde{\mathbf{v}}_{n, k}$ will determine their sign-changes.

In this proof, the $m$-th entry of $\mathbf{v}_{n, k}$ is denoted by $\mathbf{v}_{n, k}(m)$, where $m=0,1, \ldots, 2^{n}-1$. Because the first and last entries of $v_{n, k}$ will become the central two entries in $\hat{\mathbf{v}}_{n, k}$ and $\overline{\mathrm{v}}_{n, k}$, their signs should be considered to determine the sign-changes of $\hat{v}_{n, k}$ and $\hat{\mathbf{v}}_{n, k}$. All the combinations of signs for the first and last entries are shown in the first two columns of Table 1. The sign of $v_{n, k}(0)$ will be equal to those of $\hat{\mathbf{v}}_{n, k}(N)$ and $\tilde{\mathbf{v}}_{n, k}(N-1)$. The sign of $\mathbf{v}_{n, k}(N-1)$ will be equal to those of $\hat{\mathbf{v}}_{n, k}(N-1)$ and $\tilde{\mathbf{v}}_{n, k}(N)$.

The different signs in $\hat{v}_{n, k}(N-1)$ and $\hat{v}_{n, k}(N)$ will cause an additional sign-change for $\hat{\mathbf{v}}_{n, k}$. Like in the $\hat{\mathbf{v}}_{n, k}$ case, the overall sign-changes in $\tilde{\mathbf{v}}_{n, k}$ can also be determined. In Table 1, it has been shown that the sign-changes in $\hat{\mathbf{v}}_{n, k}$ and $\tilde{\mathbf{v}}_{n, k}$ are $2 k$ and $2 k+1$. One is with $2 k$ sign-changes, and the other will have $2 k+1$ sign-changes.

Proposition $6 N=2^{n}$. The numbers of sign-changes in $\mathrm{v}_{n, k}(k=0 \ldots, N-1)$ are from 0 to $N-1$. 
Proof : The proof of this proposition is by induction method. To begin with, the case $n=1$ is checked. We can find that the initial two vectors in Eq.(12) are with none and one sign-changes, respectively. Moreover, it is assumed that the case for $n=m$ is satisfied. For $n=m+1$, we can find that the numbers of sign-change are from 0 to $2 N-1$ by Proposition 5 .

Proposition 7 The absolute values in the entries of $\mathbf{v}_{n, k}$ are only the power of $(\sqrt{2}-1)^{m}$, where $m=0,1, \ldots, n$.

Proof : The proof of this proposition is trivial and directly obtained by Propositions 1 and 2.

Example 1:

Here we have an example for the Hadamard eigenvectors and eigenvalues. $N=8=2^{3}$. The initial two vectors for the Hadamard eigenvectors are $v_{1,0}$ and $v_{1,1}$, which are shown in $\mathrm{Eq}(12)$. The Hadamard eigenvectors $\mathbf{v}_{2, k}(k=0,1,2,3)$ are computed from $v_{1,0}$ and $v_{1,1}$, and they are shown in Table 2. Moreover, the Hadamard eigenvectors $\mathbf{v}_{3, k}$ can also be computed from $\mathrm{v}_{n, k}$. Table 3 shows the eigenvectors $\mathbf{v}_{3, k}$,

Because any linear combination of the eigenvectors corresponding to the same eigenvalues is still an eigenvector, the Hadamard eigenvectors obtained by propositions 1 and 2 are not the unique solution. The solution computed by our recursive algorithm is the same as that of [8]. Our recursive algorithm has the advantage that the numbers of sign-changes in the generated Hadamard eigenvectors can be evaluated and proved.

\section{Discrete fractional Hadamard transform}

Similar to development of DFRFT. The eigen decomposition of Hadamard transform will be used for the definition of discrete fractional Hadamard transform.

It must be noted that the eigenvectors obtained by Propositions 1 and 2 are not normalized. These eigenvectors should be normalized in kernel construction for the energy preserving

$$
\mathbf{Z}_{k}=\frac{\mathbf{v}_{n, k}}{\left\|\mathbf{v}_{n, k}\right\|}
$$

Thus the eigen decomposition of Hadamard transform using the Hadamard eigenvectors generated by Propositions 1 and 2 can be written as follows:

$$
\mathbf{H}_{n}=\sum_{k=0}^{2^{n}-1} e^{-j k \pi} \mathbf{Z}_{k} \mathbf{Z}_{k}^{T}
$$

In the development of DFRFT, the $k$-th order DFT Hermite eigenvectors are assigned to have the eigenvalues $e^{-j k \alpha}[3][4]$. Similar to the development of DFRFT, the normalized Hadamard eigenvectors obtained by propositions 1 and 2 with $k$ sign-changes are assigned to the eigenvalue $e^{-j k \alpha}$. Thus the definition of discrete fractional Hadamard transform is written as:

$$
\mathbf{H}_{n, \alpha}=\sum_{k=0}^{2^{n}-1} e^{-j k \alpha} \mathbf{Z}_{k} \mathbf{Z}_{k}^{T}
$$

It is easy to check that the fractional Hadamard transform will become an identity operation for $\alpha=0$, and a traditional discrete Hadamard transform for $\alpha=\pi$.

Example 2:

In this example, we will compute the discrete fractional
Hadamard transforms for an impulse signal with width 64 $(x(n)=1$, when $n=0 ; x(n)=0,1 \leq n \leq 63)$. Fig.1 shows the discrete fractional Hadamard transform outputs of this impulse signal. While $0<\alpha<\frac{\pi}{2}$, the peaks become wider as $\alpha$ grows larger. If $\alpha=\frac{\pi}{2}$, the output is two peaks. When $\frac{\pi}{2}<\alpha<\pi$, the ripples are denser as $\alpha$ is closer to $\pi$. Finally, the output becomes a DC when $\alpha=\pi$.

\section{Properties of discrete fractional Hadamard transform}

- Angle additivity

$$
\mathbf{H}_{n, \alpha} \mathbf{H}_{n, \beta}=\mathbf{H}_{n, \alpha+\beta}
$$

- Unitary

$$
\mathbf{H}_{n,-\alpha}=\mathbf{H}_{n, \alpha}^{*}=\left(\mathbf{H}_{n, \alpha}\right)^{-1}
$$

- Periodicity

The discrete fractional Hadamard transform will reduce to the classical Hadamard transform for $\alpha=\pi$.

$$
\mathbf{H}_{n, \alpha+2 \pi}=\mathbf{H}_{\boldsymbol{n}, \boldsymbol{\alpha}}
$$

- Symmetric

$$
\mathbf{H}_{n, \alpha}(a, b)=\mathbf{H}_{n, \alpha}(b, a)
$$

\section{Conclusions}

In this paper, the discrete fractional Hadamard transform based upon the same idea of DFRFT is proposed. Most of the properties of DFRFT can be preserved in the discrete fractional Hadamard transform. The proposed discrete fractional Hadamard transform can provide intermediate outputs for the signals to its Hadamard transform, and it will be useful in signal analysis.

\section{REFERENCES}

[1] L. B. Almeida, "The fractional Fourier transform and time-frequency representation," IEEE Trans. Signal Process., vol. 42, pp. 3084-3091, Nov. 1994.

[2] B. Santhanarn and J. H. McClellan, "The discrete rotational Fourier transform," IEEE Trans. Signal Process., vol. 42, pp. 994-998, April 1996.

[3] S. C. Pei and M. H. Yeh, "Discrete fractional Fourier transform," in Proceeding of IEEE International Symposium on Circuits and Systems, pp. 536-539, May 1996.

[4] S. C. Pei, M. H. Yeh, and C. C. Tseng, "Discrete fractional Fourier transform based on orthogonal projection." will appear in IEEE Trans. Signal Processing.

[5] A. K. Jain, Fundamentals of digital image processing. Prentice-Hall International Inc., 1989.

[6] S. C. Pei, C. C. Tseng, M. H. Yeh, and J. J. Shyu, "Discrete fractional Hartley and Fourier transforms," IEEE Trans. Circuit and System, Part II, vol. 45, pp. 665-675, 1998.

[7] H. M. Ozaktas, O. Arikan, M. A. Kutay, and G. Bozdagi, "Digital computation of the fractional Fourier transform," IEEE Trans. on Signal Process., vol. 44, pp. 2141-2150, Sept. 1996.

[8] R. Yarlagadda, "A note on the eigenvectors of Hadamard matrices of order $2^{n}$ " Linear Algebra and Its Applications, vol. 45, pp. 43-53, 1982. 


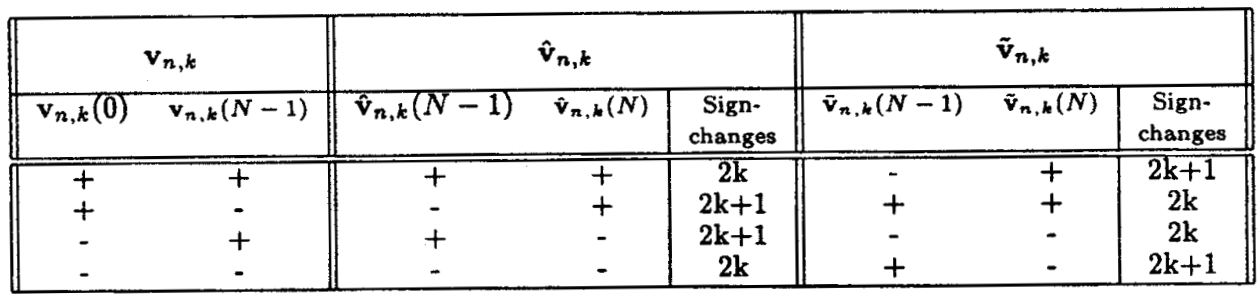

Table 1. The number of sign-changes in the constructed Hadamard eigenvector

\begin{tabular}{|c|c|c|c|}
\hline & Eigenvalue & Eigenvector & Order $2^{n+1}$ \\
\hline$\hat{\mathbf{v}}_{1,0}$ & 1 & $1,-1+\sqrt{2},-1+\sqrt{2}, 3-2 \sqrt{2}$ & $\mathbf{v}_{2,0}$ \\
\hline $\overrightarrow{\mathbf{v}}_{1,0}$ & -1 & $1-\sqrt{2}, 3+2 \sqrt{2}, 1,-1+\sqrt{2}$ & $\mathbf{v}_{2,1}$ \\
\hline$\hat{\mathbf{v}}_{1,1}$ & -1 & $1-\sqrt{2}, 1,-3+2 \sqrt{2},-1+\sqrt{2}$ & $\mathbf{v}_{2,3}$ \\
\hline$\tilde{\mathbf{v}}_{1,1}$ & 1 & {$[3-2 \sqrt{2}, 1-\sqrt{2}, 1-\sqrt{2}, 1$} & $\mathbf{v}_{2,2}$ \\
\hline
\end{tabular}

Table 2. An example for the Hadamard eigenvectors, $N=4$

\begin{tabular}{|c|c|c|c|}
\hline & Eigenvalue & Eigenvector & Order $2^{n+1}$ \\
\hline$\hat{\mathbf{v}}_{2,0}$ & 1 & $1,-1+\sqrt{2},-1+\sqrt{2}, 3-2 \sqrt{2},-1+\sqrt{2}, 3-2 \sqrt{2}, 3-2 \sqrt{2},-7+5 \sqrt{2}$ & $v_{3,0}$ \\
\hline$\tilde{\mathbf{v}}_{\mathbf{2}, 0}$ & -1 & $1-\sqrt{2},-3+2 \sqrt{2},-3+2 \sqrt{2}, 7-5 \sqrt{2}, 1,-1+\sqrt{2},-1+\sqrt{2}, 3-2 \sqrt{2}$ & $\mathbf{v}_{3,1}$ \\
\hline$\hat{\mathbf{v}}_{2,1}$ & -1 & $1-\sqrt{2},-3+2 \sqrt{2}, 1,-1+\sqrt{2},-3+2 \sqrt{2}, 7-5 \sqrt{2},-1+\sqrt{2}, 3-2 \sqrt{2}$ & $\mathbf{v}_{3,3}$ \\
\hline$\tilde{\mathbf{v}}_{2,1}$ & 1 & $3-2 \sqrt{2},-7+5 \sqrt{2}, 1-\sqrt{2},-3+2 \sqrt{2}, 1-\sqrt{2},-3+2 \sqrt{2}, 1,-1+\sqrt{2}$ & $\mathbf{v}_{3,2}$ \\
\hline$\hat{\mathbf{v}}_{2,2}$ & 1 & $3-2 \sqrt{2}, 1-\sqrt{2}, 1-\sqrt{2}, 1,-7+5 \sqrt{2},-3+2 \sqrt{2},-3+2 \sqrt{2},-1+\sqrt{2}$ & $\mathbf{v}_{3,4}$ \\
\hline$\tilde{\mathbf{v}}_{2,2}$ & -1 & {$[7-5 \sqrt{2}, 3-2 \sqrt{2}, 3-2 \sqrt{2}, 1-\sqrt{2}, 3-2 \sqrt{2}, 1-\sqrt{2}, 1-\sqrt{2}, 1]$} & $\mathbf{v}_{3,5}$ \\
\hline$\hat{\mathbf{v}}_{2,3}$ & -1 & {$[1-\sqrt{2}, 1,-3+2 \sqrt{2},-1+\sqrt{2},-3+2 \sqrt{2},-1+\sqrt{2}, 7-5 \sqrt{2}, 3-2 \sqrt{2}]$} & $\mathbf{v}_{3,7}$ \\
\hline$\overline{\mathbf{v}}_{2,3}$ & 1 & $3-2 \sqrt{2}, 1-\sqrt{2},-7+5 \sqrt{2},-3+2 \sqrt{2}, 1-\sqrt{2}, 1,-3+2 \sqrt{2},-1+\sqrt{2}$ & $\mathbf{v}_{3,6}$ \\
\hline
\end{tabular}

Table 3. An example for the Hadamard eigenvectors, $N=8$
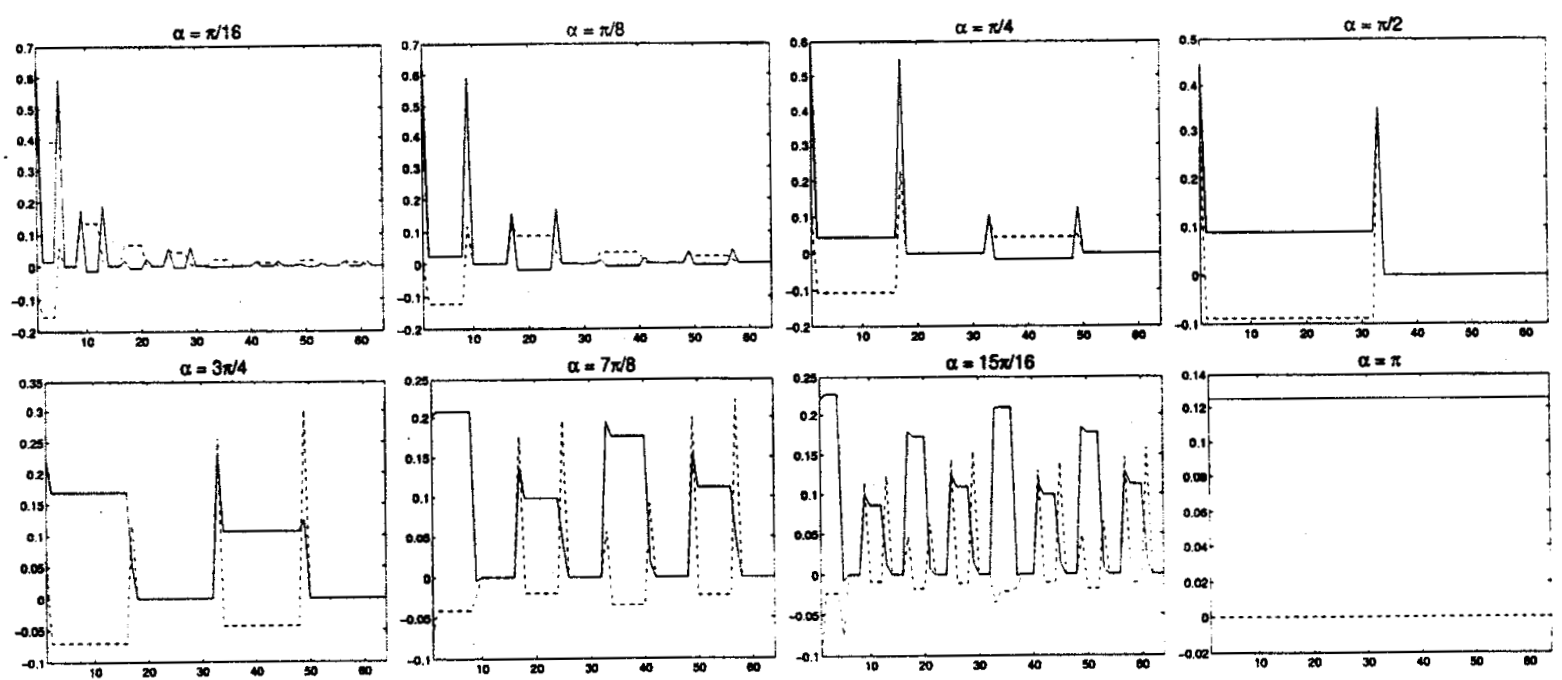

Figure 1. The discrete fractional Hadamard transform of an impulse signal 